\title{
THE SECOND BYURAKAN SURVEY
}

\author{
J. STEPANIAN \\ Special Astrophysical Observatory \\ Nizhnij Arkhyz \\ Karachaevo-Cherkessk Republic \\ 357147 Russia
}

\begin{abstract}
On the basis of the Second Byurakan Survey (SBS), we have produced a new complete sample of bright QSOs.

The observations were obtained using the 1.5, 3 and 4 degree objective prisms with the 40-52 inch Schmidt telescope at the Byurakan Observatory. The total sky coverage area of SBS is 1000 square degrees from R.A. 07h $40 \mathrm{~m}-17 \mathrm{~h} 15 \mathrm{~m}$ and Dec. $+49-+61$.

In all, in SBS about 3000 - 1600 stellar objects have been selected, and about 1400 galaxies down to a limiting magnitude $B \sim 19.5$.

290 QSO and 67 Sy galaxies are spectroscopically confirmed.

The estimate of surface density of SBS QSOs in the magnitude range $15.5<$ B $<17.2$ and redshift distribution of SBS QSOS and Sy galaxies are given.
\end{abstract}

\section{Introduction}

Wide-field sky surveys have proved essential tools for statistical astronomy, based mainly on Schmidt photographic plate surveys. In the last 30 years, from the discovery of the quasars by M. Schmidt, a large army of astronomers all over the world have discovered about 6500 QSOs. Practically all telescopes in the world have been used for searching the QSOs. Now the deepest QSO survey has reached up to the $23 \mathrm{mag}$, the most distant QSO has a redshift of $z=4.897$.

Up to 1985 the QSOs with highest redshift were discovered using radio surveys; from 1985 until now the highest redshift QSOs were discovered only from optical surveys.

Nevertheless, of 6500 QSOs discovered today, the number of bright QSOs are only a few hundred, complete samples contain only a few dozen. The total number of bright QSOs which is available from statistics from complete samples consists of about $\sim 100$ objects brighter than $B<17.0$, and $~ 200$ objects brighter than $B<17.5$.

The SBS survey contains $\sim 60$ new QSOs brighter than B $<17.0$ and $\sim 140$ new QSOs brighter than B $<17.5$, so more than $50 \%$ of all known bright QSOs (complete samples) will be added from the SBS survey.

In order to get a better understanding of the physics, evolution and spacetime distribution of QSOs, it is still necessary to increase the number of known objects, especially bright QSOs. Several projects aimed at mapping large fractions of the sky or even the whole sky are now under way. 


\section{The Second Byurakan Survey}

The first complete sample of AGN was produced from the Markarian survey (Markarian 1967), who first used a thin objective prism for the selection of peculiar extragalactic objects. Now this survey has the name FBS (the First Byurakan Survey). Ten years later a similar survey was begun in Cerro-Tololo (Smith 1976), while grism observations were made at KPNO in 1970 (Hoag 1970).

The Second Byurakan objective prism survey started in 1974 and finished in 1991. A first list of SBS objects was published in 1983 (Markarian \& Stepanian 1983). In total, we have published seven lists (Markarian \& Stepanian 1984; Markarian, Stepanian \& Erastova 1985, 1986; Stepanian, Lipovetsky \& Erastova 1988, 1990). SBS is the continuation of the Markarian survey to fainter magnitude.

The observations were obtained using a 1.5, 3 and 4 degree objective prism with the 40-52 inch Schmidt telescope at the Byurakan observatory. The method of selection was visual, i.e. scanned by eye. The criteria of selection: strong UV continuum, emission lines, energy distribution in the spectra.

Here, we briefly describe the SBS survey, which is now practically finished.

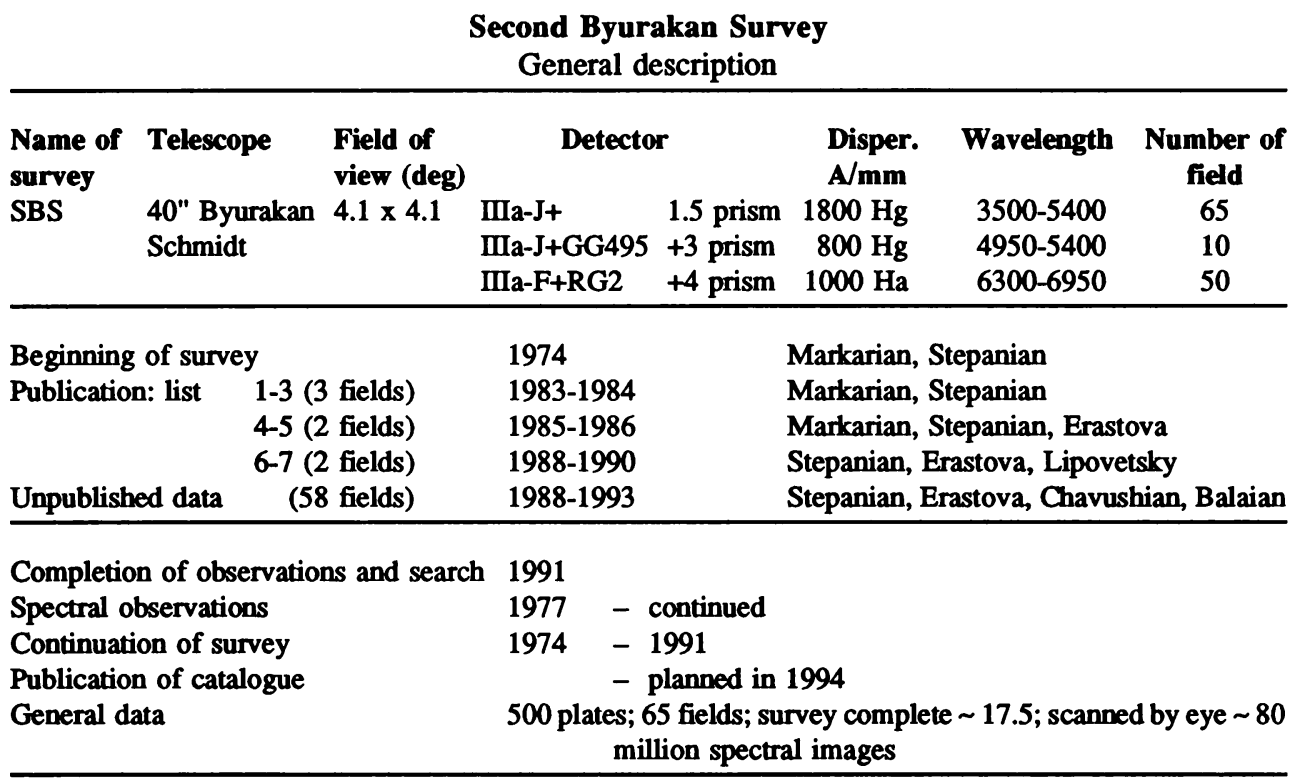

Region of sky

Limiting magnitude $\mathrm{m}(\mathrm{pg})$

Total area (sq, deg)

Total number of objects
R.A. 07 40-17 15, Dec. +49 00-61 00

$\sim 19.5$

$\sim 1000$

$\sim$ 3000; stellar objects: $\quad \sim 1600$

non stellar objects: $\sim 1400$ 


$$
\begin{aligned}
& \text { Present status slit spectra: } \quad \sim 1700(\sim 50 \%) \\
& \text { (1993) Stellar objects: } \quad \sim 1100(\sim 70 \%) \text {; QSOs }-290 \\
& \text { stars }-810 \\
& \text { Nonstellar objects: } \sim 600(\sim 45 \%) \quad \text { Sy galaxies } 67, \text { BCDG } \sim 150 \\
& \begin{array}{lll}
\text { Photometry: } & \sim 120(\sim 4 \%) & \begin{array}{l}
\text { Other types of galaxies } \sim 3 \\
\text { QSOs } \sim 90, \text { Galaxies } \sim 30
\end{array}
\end{array}
\end{aligned}
$$

Main goal: - to expand Markarian survey as deep as possible

- to produce a complete sample of bright QSOs in the mag. range 15.5 - 17.5

- to produce a complete sample of faint Markarian galaxies in the mag. range 15.5 - 17.5

- to study the large-scale structure of the Metagalaxy, large-scale distribution of QSOs, faint Markarian galaxies, and other types of selected objects, clustering of QSOs, etc.

Results of spectral observations

\begin{tabular}{lcccccc}
\hline $\begin{array}{l}\text { m(pg) } \\
\text { mag. }\end{array}$ & $\begin{array}{c}N \\
\text { total }\end{array}$ & $\begin{array}{c}N \\
\text { obs. }\end{array}$ & $\begin{array}{c}\text { Not obs. } \\
\text { total }\end{array}$ & $\begin{array}{c}\text { N (QSO) } \\
\text { z }<2.2\end{array}$ & z > 2.2 \\
\hline$<16.0$ & 188 & 188 & - & 6 & 6 & - \\
$<16.5$ & 332 & 332 & - & 18 & 18 & - \\
$<17.0$ & 548 & 540 & 8 & 60 & 56 & 4 \\
$<17.5$ & 842 & 710 & 132 & 136 & 125 & 11 \\
$<19.5$ & 1600 & 1100 & 500 & 290 & 259 & 31 \\
\hline
\end{tabular}

In Table 1 we collected together the data for a few bright quasar surveys. Some of these are finished, they are well known (BQS, LBQS), others now continuing (MBQS, CASE), while others are the new surveys (EMS, HQS).

As a rule most surveys are aimed at producing complete samples of QSOs in the investigated areas, which are limited by magnitude or by area.

It can be seen that there is a big lack between $\mathrm{BQS}$ and other surveys, by investigated area as well as by limiting mag. Up to now it has been necessary to have a survey with the area coverage of about three or four thousand square degrees, and limiting magnitude about $18.0-18.5$.

Table 1. Bright quasar surveys

\begin{tabular}{lllllll}
\hline Name & Date & Tel. Site & Type & Method & Criteria & Objects \\
\hline SBS & $1974-1991$ & $40 "$ Byurakan & Spectral & Prism Visual & UVX, Em. & QSO, UVG \\
CASE & $1983-$ cont. & 24" BS KPNO & Spectral & Prism Visual & UVX, Em. & QSO, UVG \\
LBQS & $1987-1991$ & $48 "$ UKST & Spectral & Prism Auto. & UVX, Em. & QSO \\
HQS & $1990-$ cont. & 31" Cal-Alto & Spectral & Prism Auto. & UVX, Em. & QSO \\
\hline BQS & $1973-1983$ & 18" Palomar & Photom. & UB Visual & Two-Col. & QSO, BS \\
MBQS & $1981-$ cont. & 48" Palomar & Photom. & UBV Visual & Three-Col. & BS \\
EMS & 1991-cont. & $48 "$ UKST & Photom. & UBVRI Auto. & Multi-Col. & QSO \\
\hline
\end{tabular}


Table 1 cont. Comparative characteristics

\begin{tabular}{lccccl}
\hline Name & Area (deg) & $\mathbf{m}(\mathbf{c o m p l})$ & $\mathrm{N}(\mathrm{QSO})$ & $\mathrm{z}(\max )$ & \multicolumn{1}{c}{ Authors } \\
\hline BQS & 10714 & 16.12 & 92 & 2.2 & Schmidt \& Green 1983 \\
EMS & 330 & 16.5 & 8 & $1.3:$ & Goldschmidt et al. 1992 \\
SBS & 1000 & $17.2:$ & $120:$ & 3.2 & Stepanian (this work) \\
MBQS & 108.6 & 17.65 & 32 & 2.2 & Mitchell et al. 1984 \\
LBQS & 453.8 & 18.41 & 997 & 3.4 & Foftz et al. 1993 \\
\hline
\end{tabular}

In the middle of the 70s we undertook the SBS survey. Unfortunately because of different reasons SBS was stopped in 1991, and we were able to investigate only about $~ 1000$ sq. deg.

Now the pretender of such type of survey remains in the form of CASE, EMS and HQS.

Table 2. Surface density of bright QSOs*

\begin{tabular}{|c|c|c|c|c|c|c|c|c|c|c|}
\hline \multirow[t]{2}{*}{ B } & \multicolumn{2}{|c|}{ BQS } & \multicolumn{2}{|c|}{ EMS } & \multicolumn{2}{|c|}{ MBQS } & \multicolumn{2}{|c|}{ LBQS } & \multicolumn{2}{|c|}{ SBS } \\
\hline & $\mathbf{n}$ & d & $\mathbf{n}$ & d & $\mathbf{n}$ & d & $\mathbf{n}$ & d & $\mathbf{n}$ & d \\
\hline$<15.0$ & 11 & 0.0010 & & & & & & & & \\
\hline$<15.2$ & 18 & 0.0017 & & & & & & & & \\
\hline$<15.4$ & 23 & 0.0021 & - & - & & & & & 1 & 0.001 \\
\hline$<15.5$ & & & 1 & 0.003 & - & - & & & 2 & 0.002 \\
\hline$<15.6$ & 35 & 0.0033 & 2 & 0.006 & 1 & 0.0092 & - & - & 2 & 0.002 \\
\hline$<15.8$ & 36 & 0.0036 & 3 & 0.009 & 1 & 0.0092 & - & - & 4 & 0.004 \\
\hline$<16.0$ & 44 & 0.0054 & 4 & 0.012 & 1 & 0.0092 & 1 & 0.0022 & 6 & 0.006 \\
\hline$<16.2$ & 26 & 0.0068 & 6 & 0.018 & 2 & 0.0184 & 2 & 0.0044 & 9 & 0.009 \\
\hline$<16.4$ & 8 & 0.014 & 8 & 0.024 & 4 & 0.0368 & 3 & 0.0066 & 15 & 0.015 \\
\hline$<16.5$ & & & 9: & $0.027:$ & 5 & 0.0460 & 3 & 0.0066 & 20 & 0.020 \\
\hline$<16.6$ & & & & & 7 & 0.0644 & 4 & 0.0088 & 24 & 0.024 \\
\hline$<16.8$ & & & & & 9 & 0.0828 & 8 & 0.0177 & 38 & 0.038 \\
\hline$<17.0$ & & & & & 11 & 0.101 & 18 & 0.040 & 57 & 0.057 \\
\hline$<17.2$ & & & & & 13 & 0.120 & 32 & 0.070 & 75 & 0.075 \\
\hline
\end{tabular}

*) $\mathrm{M}(\mathrm{B}) \leq 23(\mathrm{H}=50 \mathrm{~km} / \mathrm{s} \mathrm{Mpc}, \mathrm{q}(0)=0, \mathrm{z}<2.2) \mathrm{d}=\mathrm{n}(<\mathrm{B}) \mathrm{deg}-2$

\section{Results}

In total in the SBS we discovered 290 QSOs and 67 Sy galaxies. In Fig. 2 we give the redshift distribution of SBS QSOs and Sy galaxies. We predict that the complete sample of SBS QSOs will be nearly $B<17.5$ magnitude.

Spectroscopy are available for $99 \%$ and $85 \%$ for objects brighter than $B<17.0$, and $B<17.5$ respectively. Photometry is available for about $70 \%$, and $50 \%$ for objects brighter than $B<17.0$ and $B<17.5$ respectively. It can be seen from Table 2 that up to the:

$\mathrm{B}<15.5-\mathrm{BQS}$ has no competitor, it is the most powerful survey;

B $<16.0$ - if the result of Goldschmidt et al. (1992) will be confirmed in a big area, aimed at increasing the number of objects, then BQS and as well as SBS may be incomplete by a factor of 2 ; 


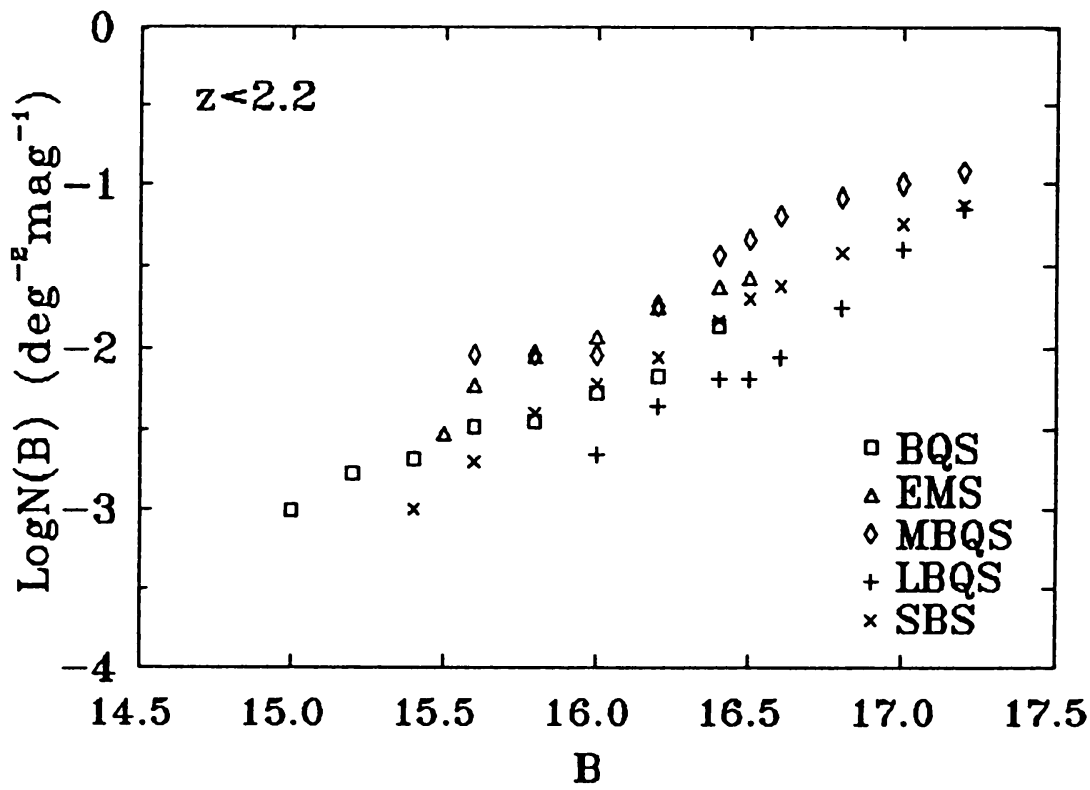

Figure 1. The number magnitude relation of SBS QSOs.
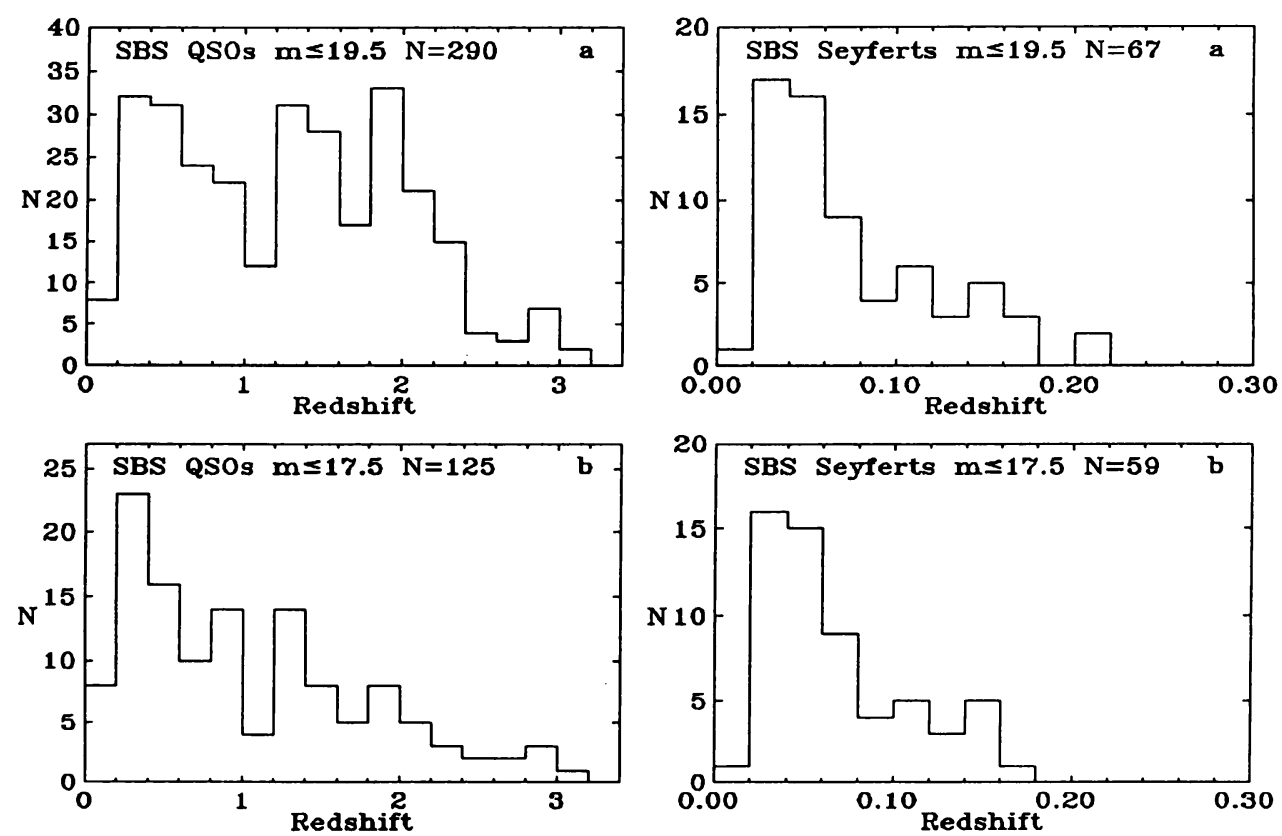

Figure 2. Redshift distribution of SBS QSOs and Sy galaxies a) full sample, b) complete sample. 
B $<16.5$ - SBS results are much lower than MBQS data (MBQS result is too high but the number of objects too small), but close to EMS data. We estimate the SBS incompleteness to be no more than a factor of 1.5;

B $<17.0$ - SBS gives the certain lowest limit to surface density.

\section{Conclusion}

- SBS is the largest continuous quasar survey with $B<17.5$;

- SBS provides a new complete sample of bright QSOs in mag range 16.5 - 17.5 and may fill this interval of magnitudes;

— SBS adds more than 50\% of all known QSO in mag range 16.5 - 17.5 available for statistics;

- SBS significantly expands the number of QSOs suitable for high dispersion investigations.

\section{Acknowledgements}

More detailed analysis of SBS data will be published after the completion of the photometry. The work is supported by a research grant from the Russian Fund of Fundamental Investigations N 93-02-17184.

\section{References}

Engels, D., Groote, D., Hagen, H.-J. and Reimers, D., 1988. In 'Proceedings of a Workshop on Optical Surveys for Quasars', eds. P. Osmer et al., p. 143.

Foftz, C.B., Chafee, F.H., Hewett, P.C., MacAlpine, G.M., Turnshek, D.A., Weymann, R.J. and Anderson, S.F., 1987. Astron. J., 94, 1423.

Goldschmidt, P., Miller, L., La Franca, F., Cristiani, S., 1992. Mon. Not. R. astron. Soc., 256, $65 p$.

Hoag, A.A., 1976. Publ. Astron. Soc. Pacific, 88, 860.

Markarian, B.E., 1967. Astrofizica, 3, 55.

Markarian, B.E. and Stepanian, J.A., 1983. Astrofizica, 19, 639.

Markarian, B.E. and Stepanian, J.A., 1984. Astrofizica, 20, 21.

Markarian, B.E. and Stepanian, J.A., 1984. Astrofizica, 20, 513.

Markarian, B.E., Stepanian, J.A. and Erastova, L.K., 1985. Astrofizica, 23, 439.

Markarian, B.E., Stepanian, J.A. and Erastova, L.K., 1986. Astrofizica, 25, 345.

Mitchell, K.J., Warnock, A.III, and Usher, P.D., 1984. Astrophys. J., 287, L3.

Pesch, P. and Sanduleak, N., 1983. Astrophys. J. Suppl., 51, 171.

Schmidt, M. and Green, R.F., 1983. Astrophys. J., 269, 352.

Smith, M.G., 1975. Astrophys. J., 202, 591.

Stepanian, J.A., Lipovetsky, V.A. and Erastova, L.K., 1988. Astrofizica, 29, 247.

Stepanian, J.A., Lipovetsky, V.A. and Erastova, L.K., 1990. Astrofizica, 32, 441. 